\title{
The Usefulness of Capsule Endoscopy for Small Bowel Tumors
}

\author{
Dae Young Cheung ${ }^{1}$, Jin Su Kim ${ }^{1}$, Ki-Nam Shim², Myung-Gyu Choi ${ }^{1}$ and Korean Gut Image Study Group \\ ${ }^{1}$ Department of Internal Medicine, College of Medicine, The Catholic University of Korea, Seoul, ${ }^{2}$ Department of Internal Medicine, Ewha \\ Womans University School of Medicine, Seoul, Korea
}

Video capsule endoscopy (VCE) has expanded the range of endoscopic examination of the small bowel. The clinical application of VCE is mainly for obscure gastrointestinal bleeding (OGIB) and small bowel tumor is one of the clinically significant diagnoses of VCE, often requiring subsequent invasive interventions. Small bowel tumors are detected with a frequency of around $4 \%$ with VCE in indications of OGIB, iron deficiency anemia, unexplained abdominal pain, and others. Protruding mass with bleeding, mucosal disruption, irregular surface, discolored area, and white villi are suggested as the VCE findings of small bowel tumor. Device assisted enteroscopy (DAE), computed tomography enteroclysis/enterography and magnetic resonance enteroclysis/enterography also have clinical value in small bowel examination and tumor detection, and they can be used with VCE, sequentially or complementarily. Familial adenomatous polyposis, Peutz-Jeghers syndrome, melanoma, lymphoma, and neuroendocrine tumor with hepatic metastasis are the high risk groups for small bowel tumors, and surveillance programs for small bowel tumors are needed. VCE and radiological imaging have value in screening, and in selected cases, DAE can provide more accurate diagnosis and endoscopic treatment. This review describes the usefulness and clinical impact of VCE on small bowel tumors. Clin Endosc 2016;49:21-25

Key Words: Small bowel; Neoplasms; Capsule endoscopy

\section{INTRODUCTION}

The small intestine is the largest organ in the body and occupies over $90 \%$ of the surface of the gastrointestinal tract. However, diagnosis of small bowel tumor is rare in practice. ${ }^{1}$ Rapid epithelial turnover with rapid intestinal transit, abundant lymphoid tissue and immunoglobulin $\mathrm{A}$ secretion, neutral $\mathrm{pH}$, and lower bacterial load are responsible in explaining the extremely low tumorigenic property of the small intestine. Only $3 \%$ to $6 \%$ of all gastrointestinal neoplasms develop in the small intestine and over 40 different histological types of tumors are diagnosed. Adenoma and mesenchymal tumors are the most common ones. Ma-

Received: January 4, 2016 Revised: January 14, 2016

Accepted: January 15, 2016

Correspondence: Ki-Nam Shim

Department of Internal Medicine, Ewha Womans University School of Medicine, 1071 Anyangcheon-ro, Yangcheon-gu, Seoul 07985, Korea

Tel: +82-2-2650-2632, Fax: +82-2-2655-2076, E-mail: shimkn@ewha.ac.kr

(cc) This is an Open Access article distributed under the terms of the Creative Commons Attribution Non-Commercial License (http://creativecommons.org/ licenses/by-nc/3.0) which permits unrestricted non-commercial use, distribution, and reproduction in any medium, provided the original work is properly cited. lignant tumors include neuroendocrine tumor, adenocarcinoma, lymphoma, and sarcoma in order of frequency. ${ }^{2}$ Skin melanoma, colorectal cancer, prostate cancer, and lung and breast cancers are the common origins of metastatic small bowel tumors.

Capsule endoscopy is a small capsule type digital camera which can be swallowed orally and takes images of the small bowel lumen. The major indications for small bowel video capsule endoscopy (VCE) are obscure gastrointestinal bleeding (OGIB), suspicious small bowel Crohn disease (CD), unexplained iron deficiency anemia (IDA), and unexplained abdominal pain. The diagnostic yield of VCE is any detectable lesion which can explain the clinical manifestation, and small bowel tumor is one of the significant diagnoses. The frequency of small bowel tumor in VCE for all indications ranges from $2.4 \%$ in European study ${ }^{3}$ to $4.3 \%$ to $9.5 \%$ in a Korean study. ${ }^{4,5}$ Guidelines for VCE in small bowel diseases are published in Korea and Europe. ${ }^{6-8}$ The clinical value of VCE for small bowel tumors are reviewed here in comparison to other small bowel examination modalities. 


\section{THE VAGUE CLINICAL PRESENTATION OF SMALL BOWEL TUMORS}

There is no definite clinical manifestation suggestive of small bowel tumor. Most clinical presentations are vague and non-specific. Therefore, most cases of small bowel tumors are unexpectedly diagnosed in the course of a thorough diagnostic workup for patients with OGIB, unexplained IDA, and unexplained abdominal pain. Obscure or overt bleeding is the most common presentation and occurs due to ulceration of tumors. Patients presenting with OGIB are proven to have small bowel tumor in around $6 \%$ of cases. ${ }^{9-12}$ Two out of 110 patients presenting with unexplained abdominal pain were revealed to have small bowel tumor. ${ }^{13}$ Nausea and vomiting can occur when obstruction develops, especially in a form of intussusception. Weight loss is an alarming sign requiring a thorough investigation, especially in elderly patients, and presenting in $30 \%$ to $50 \%$ of small bowel tumors. However, small sized tumors are mostly asymptomatic. When the signs and symptoms of small bowel tumor become obvious, it can already be in an advanced stage, and have metastasis. The vagueness of presentation can delay the diagnosis and make the prognosis less favorable in malignant tumors. In short, small bowel tumors should be listed as a possible diagnosis when patients present with OGIB, unexplained abdominal pain, nausea, and vomiting and weight loss. Suspicious findings on radiological imaging are also a significant suggestion of small bowel tumors.

High-risk group of small bowel tumors should be borne in mind. Follicular lymphoma, metastatic neuroendocrine tumor, and malignant melanoma often have small bowel involvement. ${ }^{14,15}$ The risk of small bowel adenocarcinoma is increased with long lasting small bowel CD and inherited polyposis syndromes including familial adenomatous polyposis (FAP), Peutz-Jeghers syndrome (PJS), and Lynch syndrome (hereditary non-polyposis colorectal cancer). Small bowel surveillance strategies are on the table for FAP and PJS. Celiac disease increases the risk of small bowel $\mathrm{T}$ cell lymphoma and adenocarcinoma in Western population. ${ }^{16}$ Smoking and red meat intake are related to small bowel cancer as well as colorectal cancer.

\section{THE VALUE AND PRIORITY OF VCE IN DIAGNOSIS OF SMALL BOWEL TUMORS}

Small bowel investigation is now possible and new modalities include VCE, device assisted enteroscopy (DAE), computed tomography enteroclysis/enterography (CTE), and magnetic resonance enteroclysis/enterography (MRE). Push enteroscopy and sonde endoscopy were the sole endoscopic tools in the past, but now are scarcely used in clinical practice. Small bowel series, small bowel follow-through (SBFT), and enteroclysis are radiological imagings. In the past, enteroclysis was accepted as the most accurate diagnostic tool for small bowel disease, but caused high inconvenience both for physician and patient, preventing routine clinical usage.

When OGIB and IDA are not explained otherwise, and small bowel tumors are suspected, European guidelines recommend early use of VCE. ${ }^{8}$ In OGIB, VCE yields better diagnostic outcomes than SBFT and enteroclysis, and the diagnostic yield was $50 \%$ to $75 \% .{ }^{17}$ VCE provided superior diagnostic yields to push enteroscopy, $50 \%$ to $67.2 \%$, and $20 \%$ to $28 \%$, respectively. ${ }^{18-20}$ However, because the reach of push enteroscopy is reliable up to proximal jejunum, in case of the small bowel tumor detected on VCE, and located in proximal jejunum, push enteroscopy can be employed to obtain tissue biopsies. VCE and DAE showed comparable diagnostic yields. When DAE was performed with one sided approach, VCE provided better diagnostic yield than DAE. ${ }^{21}$

The findings of small bowel tumor in VCE are not always easily interpreted. An experimental proposal by Chinese researchers recently described a scoring system to interpret VCE findings in small bowel tumors. The proposed tumor score was composed of five components: bleeding, mucosal disruption, an irregular surface, color, and white villi. These can be scored for probability of mass lesions seen at capsule endoscopy. ${ }^{22}$ This system is not perfect yet, but these factors have to be kept in mind. Contrarily, innocent bulging can be characterized as a mass with ill-defined boundaries, larger diameter than height, invisible lumen with a mass like contour, and mass image lasting less than 10 minutes. $^{23}$ MRE and CTE reported similar diagnostic yields to VCE. When MRE was performed in patients with suspicion of small bowel tumor, the overall sensitivity, specificity, and accuracy in identifying patients with small-bowel lesions were $86 \%, 98 \%$, and $97 \%$, respectively. ${ }^{24}$ In a study of small bowel diseases, including small bowel tumor, $\mathrm{CD}$, and others, the diagnostic specificity of MRE was higher than that of VCE ( 0.97 vs. $0.84, p=0.047)$, whereas sensitivity was similar ( 0.79 vs. $0.74, p=0.591) .{ }^{25}$ In a small radiological study for OGIB, CTE demonstrated better diagnostic yield than VCE, in term of sensitivity (88\% vs. $38 \%){ }^{26}$ Authors reported that, CTE detected 9 of 9 tumors but VCE only 3 of 9 tumors. This is a kind of deviated result from others, and we can assume that the small bowel tumors with exophytic growth may possibly be overlooked by VCE. Study populations and properties of small bowel tumors also affect the study outcomes, and we cannot easily determine the superiority of a single examination to another. MRE and CTE can provide the intra- and 
extra-luminal images combined with contrast enhancement and functional images. MRE and CTE may serve as the firstline diagnostic tool along with VCE for patients with OGIB and suspicion of small bowel tumors.

The detection of small bowel tumors with DAE was dependent on clinical suspicion, and was very seldom due to a general indication. In patients who were suspected of having small bowel tumors in other studies, there was a markedly high rate $(47 \%)$ of tumor diagnosis with DBE. ${ }^{27}$ In Korean studies, DAE for OGIB, detected small bowel tumors in $17.4 \%$ to $19.7 \%$ cases and small bowel tumors are common following small bowel ulcer and vascular lesion. ${ }^{9,28}$ Benign polyps are the most common (33.9\%), followed by mesenchymal tumors, lymphoma, adenocarcinoma, and metastatic cancers in sequence. ${ }^{28}$ Diagnostic concordance was $78.3 \%$ for small bowel tumor between DAE and VCE. ${ }^{28}$ while it was $7 \%$ to $20 \%$ in Japanese reports. ${ }^{12,29}$ The higher small bowel tumor detection rate of DAE over VCE must result from the patient selection difference as well as the better maneuverability. The risk of false negative results should be always taken into account in VCE. ${ }^{30}$ VCE may easily miss the tumors which are located in duodenum or proximal jejunum due to rapid transit. The subepithelial tumors with intact overlying mucosa also can be missed with VCE. Another critical advantage of DAE over VCE is the possibility of endoscopic treatment, mainly polypectomy. Patients with PJS and FAP are at high risk of small bowel polyps, and polypectomy using DBE can prevent the possibility of small bowel obstruction and the need of subsequent small bowel resections in PJS. ${ }^{31-33}$ DBE is comparably effective but less invasive than intraoperative endoscopy in patients with PJS. ${ }^{32}$ DAE, however, often fails to achieve complete small bowel examination, elicits significant discomfort, radiation exposure, and leads to complications in patients during examination. Therefore, DAE might be better employed in a highly selected group, while VCE is used initially to detect patients with possible small bowel tumors, from the larger group with OGIB. DAE should be considered in preference to VCE to confirm the diagnosis through visualization and histology, when small bowel tumors are suspected with prior imaging studies. ${ }^{8}$

The only procedure related complication of VCE is capsule retention. Capsule retention occurred in $2.5 \%$ of all cases. ${ }^{33}$ $\mathrm{CD}$ and intestinal tuberculosis often lead to luminal stenosis and capsule retention. Small bowel tumors are also one of the major diseases associated with capsule retention. However, on suspecting small bowel tumor, patency investigation before VCE is not needed. ${ }^{8}$ Capsule retention occurs only at the point of pathology and can guide the surgery.

\section{HIGH-RISK GROUP SURVEILLANCE FOR SMALL BOWEL TUMOR}

Small bowel tumor surveillance in high-risk group can result in early detection of disease and make the prognosis better. VCE as well as CTE and MRE can help in surveillance. Inherited polyposis syndromes, melanoma, lymphoma, and neuroendocrine tumor with hepatic metastasis are well known high-risk groups of small bowel tumors. Inherited polyposis syndromes including FAP and PJS are at increased risk of small bowel adenocarcinoma as well as colorectal cancer.

In patients with FAP, duodenal adenomatosis commonly develops and increases the risk of cancer with age. The cumulative lifetime risk of duodenal adenomatosis is $88 \%$ and the cumulative incidence of cancer was $18 \%$ at 75 years of age. ${ }^{34}$ Spigelman staging is a well known predictor system for malignant changes in duodenal adenomatosis, and consists of the number of polyps, size, histology, and degree of dysplasia. ${ }^{35}$ Spigelman stage IV on esophagogastroduodenoscopy (EGD) predicts a cancer risk of $33 \%$ at 75 years of age. Surveillance of the proximal small bowel in FAP is properly performed using conventional forward viewing EGD and side-viewing duodenoscopy. ${ }^{8}$ Up to $70 \%$ of patients with FAP have adenomatous polyps in jejunum and ileum; however, cancer has been rarely reported. VCE as well as CTE or MRE can be considered to detect polyps in the rest of the small bowel, but the clinical relevance is not established yet.

PJS is a hereditary polyposis syndrome and patients with PJS often present polyp related symptoms from an early age. Intussusceptions and anemia are common in childhood and need surgery. Later in life, polyps can progress to cancer, and the relative risk of small bowel cancer is about 520 (95\% confidence interval, 220 to 1,306$).{ }^{36}$ Increased risk of cancer is identified in small bowel as well as esophagus, stomach, colon, pancreas, breast, uterus, and ovary. The lifetime cumulative risk for all cancer was $93 \%$ at 64 years of age. Prophylactic polypectomy can reduce the development of polyp related complications. Screening EGD and colonoscopy are recommended, beginning at the age of 18 , with 2- to 3-year intervals. ${ }^{37}$ Small bowel surveillance is also needed to perform timely polypectomy and reduce polyp related complications and future risk of small bowel cancer. VCE is recommended as an adequate surveillance method as well as MRE in European guidelines. ${ }^{8}$ VCE has a better sensitivity in detecting small bowel polyps than SBFT and MRE, especially for small polyps. VCE and MRE both equally detect larger polyps $>1.0$ $\mathrm{cm} .{ }^{38}$ MRE has an advantage in polyp localization and accurate size measurement. Polyps $>1.5 \mathrm{~cm}$ is better defined with MRE, and false negative results may occur with VCE. ${ }^{39}$ Small bowel screening using VCE is recommended to be preformed 
every 3 years if polyps are found at the initial examination, from age of 8 years, or earlier if the patient is symptomatic. ${ }^{40}$ If few or no polyps are found at the initial examination, screening should commence again at the age of 18 years. ${ }^{40} \mathrm{DAE}$ is more accurate in detecting small bowel polyps than VCE, but DAE may be better employed following VCE for the purpose of endoscopic treatment. Polyp size is the most important factor for polyp related complications. Polyps $>1.5 \mathrm{~cm}$ often result in intussusception and are the indication for DAE polypectomy. However, when there is a concern about safe polypectomy for a larger polyp or which is in an unreachable position with DAE, intraoperative enteroscopy could be considered.

Lynch syndrome is another well-known condition associated with an increased risk of small bowel cancer. Lynch syndrome is caused by a germline mutation in one in the mismatch repair (MMR) genes MLH1, MSJ2/Epcam, MSH6, or PMS2. Asymptomatic mutation carriers are recommended to undergo surveillance colonoscopy every 1 to 2 years, starting from the age of 20 to 25 years. The estimated lifetime risk for small bowel cancer is $4.2 \%{ }^{41}$ and is similar to that of colorectal cancer in the general population. Because the small bowel cancer localization is almost even in duodenum, jejunum, and ileum, screening with conventional EGD is recommended to detect both stomach and small bowel cancer since small bowel cancer localization is almost even in duodenum, jejunum and ileum, In a recent study with VCE, the prevalence of small-bowel tumor in an asymptomatic mutation carrier of Lynch syndrome was 1.5\%. However, all tumors were located in the duodenum and within reach of conventional EGD. ${ }^{42}$

\section{CONCLUSIONS}

Small bowel tumors are diagnosed in about $4 \%$ of cases of OGIB, and other small bowel study indications. Clinical suspicion of small bowel tumor can raise the diagnostic yields in VCE and DAE. VCE and MRE/CTE can serve as proper screening tools for small bowel tumors in patients presenting with small bowel symptoms. DAE might be better employed for endoscopic treatment or biopsy after VCE or MRE/CTE with impression of small bowel tumor. Highrisk group of small bowel tumors includes patients with FAP, PJS, Lynch syndrome, and lymphoma and skin melanoma. VCE as well as MRE/CTE are promising in small bowel surveillance.

\section{Conflicts of Interest}

The authors have no financial conflicts of interest.

\section{REFERENCES}

1. Siegel RL, Miller KD, Jemal A. Cancer statistics, 2015. CA Cancer J Clin 2015;65:5-29.

2. Goodman MT, Matsuno RK, Shvetsov YB. Racial and ethnic variation in the incidence of small-bowel cancer subtypes in the United States, 1995-2008. Dis Colon Rectum 2013;56:441-448.

3. Rondonotti E, Pennazio M, Toth E, et al. Small-bowel neoplasms in patients undergoing video capsule endoscopy: a multicenter European study. Endoscopy 2008;40:488-495.

4. Lim YJ, Lee OY, Jeen YT, et al. Indications for detection, completion, and retention rates of small bowel capsule endoscopy based on the 10year data from the Korean Capsule Endoscopy Registry. Clin Endosc 2015;48:399-404.

5. Cheung DY, Lee IS, Chang DK, et al. Capsule endoscopy in small bowel tumors: a multicenter Korean study. J Gastroenterol Hepatol 2010;25:1079-1086.

6. Park SK, Ye BD, Kim KO, et al. Guidelines for video capsule endoscopy: emphasis on Crohn's disease. Clin Endosc 2015;48:128-135.

7. Shim KN, Moon JS, Chang DK, et al. Guideline for capsule endoscopy: obscure gastrointestinal bleeding. Clin Endosc 2013;46:45-53.

8. Pennazio M, Spada C, Eliakim R, et al. Small-bowel capsule endoscopy and device-assisted enteroscopy for diagnosis and treatment of small-bowel disorders: European Society of Gastrointestinal Endoscopy (ESGE) Clinical Guideline. Endoscopy 2015;47:352-376.

9. Choi H, Choi KY, Eun CS, et al. Korean experience with double balloon endoscopy: Korean Association for the Study of Intestinal Diseases multi-center study. Gastrointest Endosc 2007;66(3 Suppl):S22-S25.

10. Hadithi M, Heine GD, Jacobs MA, van Bodegraven AA, Mulder CJ. A prospective study comparing video capsule endoscopy with double-balloon enteroscopy in patients with obscure gastrointestinal bleeding. Am J Gastroenterol 2006;101:52-57.

11. Hindryckx P, Botelberge T, De Vos M, De Looze D. Clinical impact of capsule endoscopy on further strategy and long-term clinical outcome in patients with obscure bleeding. Gastrointest Endosc 2008;68:98-104.

12. Ohmiya N, Yano T, Yamamoto H, et al. Diagnosis and treatment of obscure GI bleeding at double balloon endoscopy. Gastrointest Endosc 2007;66(3 Suppl):S72-S77.

13. Shim KN, Kim YS, Kim KJ, et al. Abdominal pain accompanied by weight loss may increase the diagnostic yield of capsule endoscopy: a Korean multicenter study. Scand J Gastroenterol 2006;41:983-988.

14. Albert JG, Fechner M, Fiedler E, et al. Algorithm for detection of small-bowel metastasis in malignant melanoma of the skin. Endoscopy 2011;43:490-498.

15. Frilling A, Smith G, Clift AK, Martin J. Capsule endoscopy to detect primary tumour site in metastatic neuroendocrine tumours. Dig Liver Dis 2014;46:1038-1042.

16. Green PH, Fleischauer AT, Bhagat G, Goyal R, Jabri B, Neugut AI. Risk of malignancy in patients with celiac disease. Am J Med 2003;115:191195.

17. Costamagna G, Shah SK, Riccioni ME, et al. A prospective trial comparing small bowel radiographs and video capsule endoscopy for suspected small bowel disease. Gastroenterology 2002;123:999-1005.

18. de Leusse A, Vahedi K, Edery J, et al. Capsule endoscopy or push enteroscopy for first-line exploration of obscure gastrointestinal bleeding? Gastroenterology 2007;132:855-862.

19. Saurin JC, Delvaux M, Gaudin JL, et al. Diagnostic value of endoscopic capsule in patients with obscure digestive bleeding: blinded comparison with video push-enteroscopy. Endoscopy 2003;35:576-584.

20. Ell C, Remke S, May A, Helou L, Henrich R, Mayer G. The first prospective controlled trial comparing wireless capsule endoscopy with push enteroscopy in chronic gastrointestinal bleeding. Endoscopy 2002;34:685-689.

21. Chen X, Ran ZH, Tong JL. A meta-analysis of the yield of capsule en- 
doscopy compared to double-balloon enteroscopy in patients with small bowel diseases. World J Gastroenterol 2007;13:4372-4378.

22. Shyung LR, Lin SC, Shih SC, Chang WH, Chu CH, Wang TE. Proposed scoring system to determine small bowel mass lesions using capsule endoscopy. J Formos Med Assoc 2009;108:533-538.

23. Girelli CM, Porta P, Colombo E, Lesinigo E, Bernasconi G. Development of a novel index to discriminate bulge from mass on small-bowel capsule endoscopy. Gastrointest Endosc 2011;74:1067-1074.

24. Masselli G, Polettini E, Casciani E, Bertini L, Vecchioli A, Gualdi G. Small-bowel neoplasms: prospective evaluation of MR enteroclysis. Radiology 2009;251:743-750.

25. Van Weyenberg SJ, Bouman K, Jacobs MA, et al. Comparison of MR enteroclysis with video capsule endoscopy in the investigation of small-intestinal disease. Abdom Imaging 2013;38:42-51.

26. Huprich JE, Fletcher JG, Fidler JL, et al. Prospective blinded comparison of wireless capsule endoscopy and multiphase CT enterography in obscure gastrointestinal bleeding. Radiology 2011;260:744-751.

27. Mitsui K, Tanaka S, Yamamoto H, et al. Role of double-balloon endoscopy in the diagnosis of small-bowel tumors: the first Japanese multicenter study. Gastrointest Endosc 2009;70:498-504.

28. Lee BI, Choi H, Choi KY, et al. Clinical characteristics of small bowel tumors diagnosed by double-balloon endoscopy: KASID multi-center study. Dig Dis Sci 2011;56:2920-2927.

29. Takano N, Yamada A, Watabe H, et al. Single-balloon versus double-balloon endoscopy for achieving total enteroscopy: a randomized, controlled trial. Gastrointest Endosc 2011;73:734-739.

30. Ross A, Mehdizadeh S, Tokar J, et al. Double balloon enteroscopy detects small bowel mass lesions missed by capsule endoscopy. Dig Dis Sci 2008; $53: 2140-2143$

31. Mönkemüller K, Fry LC, Ebert M, et al. Feasibility of double-balloon enteroscopy-assisted chromoendoscopy of the small bowel in patients with familial adenomatous polyposis. Endoscopy 2007;39:52-57.

32. Kopácová M, Bures J, Ferko A, Tachecí I, Rejchrt S. Comparison of intraoperative enteroscopy and double-balloon enteroscopy for the diagnosis and treatment of Peutz-Jeghers syndrome. Surg Endosc 2010;24:1904-1910.

33. Cheon JH, Kim YS, Lee IS, et al. Can we predict spontaneous capsule passage after retention? A nationwide study to evaluate the incidence and clinical outcomes of capsule retention. Endoscopy 2007;39:10461052.

34. Bülow S, Christensen IJ, Højen H, et al. Duodenal surveillance improves the prognosis after duodenal cancer in familial adenomatous polyposis. Colorectal Dis 2012;14:947-952.

35. Spigelman AD, Williams CB, Talbot IC, Domizio P, Phillips RK. Upper gastrointestinal cancer in patients with familial adenomatous polyposis. Lancet 1989;2:783-785.

36. Giardiello FM, Brensinger JD, Tersmette AC, et al. Very high risk of cancer in familial Peutz-Jeghers syndrome. Gastroenterology 2000;119:1447-1453.

37. Giardiello FM, Trimbath JD. Peutz-Jeghers syndrome and management recommendations. Clin Gastroenterol Hepatol 2006;4:408-415.

38. Postgate A, Hyer W, Phillips R, et al. Feasibility of video capsule endoscopy in the management of children with Peutz-Jeghers syndrome: a blinded comparison with barium enterography for the detection of small bowel polyps. J Pediatr Gastroenterol Nutr 2009;49:417-423.

39. Gupta A, Postgate AJ, Burling D, et al. A prospective study of MR enterography versus capsule endoscopy for the surveillance of adult patients with Peutz-Jeghers syndrome. AJR Am J Roentgenol 2010;195:108-116

40. Beggs AD, Latchford AR, Vasen HF, et al. Peutz-Jeghers syndrome: a systematic review and recommendations for management. Gut 2010;59:975-986.

41. Koornstra JJ, Kleibeuker JH, Vasen HF. Small-bowel cancer in Lynch syndrome: is it time for surveillance? Lancet Oncol 2008;9:901-905.

42. Haanstra JF, Al-Toma A, Dekker E, et al. Prevalence of small-bowel neoplasia in Lynch syndrome assessed by video capsule endoscopy. Gut 2015;64:1578-1583. 